\title{
Eine Abscheidungsmethode für die bei der Uranspaltung auftretenden Seltenen Erden nach dem Verfahren von Szilard und Chalmers*
}

\author{
Von Hans Götte \\ Aus dem Kaiser-Wilhelm-Institut für Chemie, Tailfingen \\ (Z. Naturforschg. 1, 377-382 [1946]; eingegangen am 15. Mai 1946)
}

\begin{abstract}
Die Seltenen Erden, lassen sich mit Hilfe eines $\mathrm{Szilard-Chalmers-Verfahrens} \mathrm{vom}$ Uran und Uran $\mathrm{X}$ trennen. Das $\mathrm{UO}_{2}$ wird als inneres Komplexsalz des Uranyldibenzoylmiethans in Lösung gẹbracht und gehalten. Da das Dibenzoylmethan auch mit den Elementen der 4. Gruppe lösliche und gegen Wasser beständige Komplexe bildet, werden diese Elemente ebenso wie das Uran in organischer Lösung gehalten und nicht mit ausgeschüttelt.

Mit Hilfe der geschilderten Abscheidungsmethode wurden ein neues Cer-Isotop von 11 Min. Halbwertszeit und ein sich aus ihm nachbildendes Praseodym von 25 Min. Halbwertszeit aufgefunden. Die $\beta$-Strahlung des gefundenen Praseodyms ist beträchtlich härter als die des Cers. Außerdem wurden kurzlebige Seltene Erd-Isotope von 3 bis 5 Min. Hwz festgestellt.
\end{abstract}

$\mathrm{D}$ er grundsätzliche Unterschied zwischen einer üblichen chemisch-analytischen Arbeit und der eines Radiochemikers besteht darin, daß für eine normale chemische Analyse beliebig viel Zeit zur Verfügung steht, während das radiochemische Arbeiten infolge der mehr oder weniger langen Lebensdauer der $\mathrm{zu}$ untersuchenden Elemente stark an die Zeit gebunden ist. Es kommt in der Radiochemie häufig darauf an, in kurzer und kürzester Zeit chemische Trennungen durchzuführen und dennoch saubere Niederschläge zu gewinnen. An Stelle der Verwendung des zeitraubenden Analysenganges muß bei der Uranspaltung häufig mit Hilfe spezifischer Reaktionen aus der Vielzahl der vorliegenden Isotope das zu untersuchende isoliert werden. Betrachtet man die Halbwertszeiten der Uranspaltprodukte, so haben sich fast überall dort Elemente kurzer Halbwertszeit nachweisen lassen, wo es möglich war, mit Hilfe weniger und schnell durchführbarer chemischer oder physikalischer Operationen ein Element sicher vom Uran, UX und den auftretenden Spaltprodukten abzutrennen. Es sei an die Isotope des Jods und Broms ${ }^{1}$, die des Strontiums und Bariums ${ }^{2}$, des Rutheniums ${ }^{3}$, die kurzlebigen Edelgase ${ }^{4}$ und die Alkalien ${ }^{5}$ erinnert. Die quantitative Abtrennung von Uran und

* Der wesentliche Inhalt dieser Arbeit wurde bereits gelegentlich einer Chemiker-Tagung am 10.2. 44 vorgetragen und ist im Auszug in der Angew. Chemie (Neue Folge „Die Chemie“) 58, 46 [1945] erschienen.

1 O. Hahn u. F. Straßmann, Naturwiss. 28, 817 [1940].

2 O. Hahn u. F. Straßmann, Z. Physik 121, 729; Naturwiss. 30, 324 [1942].
UX ist bei diesen Untersuchungen besonders wichtig, weil bei Zählrohrmessungen, wie sie bei den in Deutschland zur Verfügung stehenden Strahlenquellen meistens üblich sind, die Aktivität des UX die gewonnenen künstlichen Aktivitäten weit überwiegt.

Um die bei der Uranspaltung anfallenden Seltenen Erden und das Yttrium direkt aus dem Uran abscheiden und aus diesem Gemisch einzelne Elemente, wie z. B. Lanthan oder Cer, isolieren zu können, wird bei Verwendung der gewöhnlichen analytischen Operationen eine Zeit benötigt, die es unmöglich macht, nach kurzlebigen Substanzen zu suchen. Erst die von $\mathrm{Hahn}$ und Straßmann entwickelte Methode ${ }^{6}$, die Folgeprodukte der Edelgase entweder mit Hilfe emanierender Uranpräparate oder nach fraktionierter Adsorption der Edelgase an Kohle abzutrennen, hat zur Auffindung von Lanthan- und Yttrium-Isotopen geführt, die 10-15 Min. Halbwertszeit haben. Primär bei der Spaltung auftretende Seltene Erden können nach diesem Verfahren allerdings nicht festgestellt werden.

Um die primären wie auch die sekundären Yttriumund Seltenen Erd-Isotope in kürzester Zeit abtrennen zu können, kann man sich einer auf den Gedanken von L.Szilard und T.A. Chalmers ${ }^{7}$ zurückgehenden Methode bedienen.

Nach dem Verfahren von Szilard und Chalmers werden von der großen Menge eines inaktiven Elementes die z. B. durch Neutroneneinfang in unwägbaren Mengen

${ }^{3}$ W. S e e l m a n n-E. u. H. J. B or n, Naturwiss. 31, 420 [1943].

4 W. Se elm ann-E. u. H. J. B orn, Naturwiss. 31, 59 [1943].

5 O. Hahn u. F. Straßmann, Naturwiss. 28, 54 [1940].

6 O. Hahn u. F.Stra $\beta$ mann, l. c.

7 Nature [London] 134, 462 [1934]. 
sich bildenden radioaktiven Isotope abgetrennt, indem man das zu bestrahlende Element in organische Bindung und organische Lösung bringt. Die durch kernphysikalische Prozesse entstehenden radioaktiven Isotope liegen zunächst meist als Ionen vor und lassen sich daher, wie O. Erbacher und K. Philipp ${ }^{8}$ gezeigt haben, aus dem organischen Medium mit Wasser ausschütteln oder an Kohle adsorbieren. Es findet bei diesem Verfahren also eine Anreicherung und gleichzeitige Abtrennung der neu gebildeten Isotope von den vorhandenen inaktiven statt.

Im Falle der Abtrennung von Uranspaltprodukten muß das Uran chemisch stabil in organische Lösung gebracht werden, damit beim Ausschütteln nur Spaltprodukte und kein Uran in das Wasser übergehen. Damit wäre die Abtrennung der großen Uranmenge von den in unwägbaren Mengen gebildeten Spaltprodukten möglich. Gleichzeitig darf aber auch das sich im Gleichgewicht mit dem Uran befindende oder sich aus ihm nachbildende UX nicht in das Wasser gehen, obwohl es ja aus dem Uran ebenfalls durch radioaktiven Zerfall entsteht und so zunächst auch als Ion vorliegt. Dies ist nur dann möglich, wenn die neu entstehenden oder vorhandenen UX-Ionen ebenfalls als Komplex im organischen Lösungsmittel festgehalten werden. Auf dieselbe Weise sollten auch andere bei der Spaltung auftretende, nur mit Zeitverlust abzutrennende Elemente aus der wäßrigen Phase ferngehalten werden, um die Zahl der chemischen Operationen, die zur Reinabscheidung irgendeines Spaltproduktes oder einer Gruppe davon führen, möglichst gering zu halten. Dies könnte sich durch Hinzufügen geeigneter organischer Komplexbildner erreichen lassen, die mit den zurückzuhaltenden Spaltelementen einen beständigen und in organischen Lösungsmitteln löslichen Komplex bilden. Der Idealfall wäre der, in dem nur das zu untersuchende Element zur Ausschüttelung käme.

Unter den Uranverbindungen sind solche mit direkter U-C-Bindung mit Ausnahme des Urancarbids nicht bekannt. Es mußte daher unter den Innerkomplexsalzen nach geeigneten Verbindungen gesucht werden. Hier erwiesen sich Komplexsalze der 1.3-Diketone als brauchbar. Schon das einfachste Diketon, das Acetylaceton, bildet leicht eine Uranverbindung, die bereits die für die ganze Reihe der Körper charakteristische rotgelbe Farbe zeigt. Jedoch ist das Uranylacetylaceton noch ungeeignet für ein Szilard-Chalmers-Verfahren, da. es ziemlich löslich in Wasser ist. Das Uranyl-benzoylaceton dagegen, auf dessen mögliche Verwendung für ein Szilard-Chalmers-Verfahren schon K. Starke hingewiesen hat ${ }^{\text {, }}$ oder die Innerkomplexverbindung des Uranylions mit dem Benzoylessigester sind bereits in Wasser unlöslich. Sie dürften für ein Szil ar d-Chalmers-Verfahren geeignet sein, wurden jedoch nicht näher untersucht.

Eine Uranverbindung, die den obengenannten Forderungen bei der Abtrennung der Seltenen Erden und des ihnen ähnlichen Elements Yttrium sicher genügend entspricht, ist das Uranyl-diben-

8 Z. physik. Chem. Abt. A 176, 169 [1936]. zoylmethan. Auf die Eignung dieser Verbindung für die Abtrennung der Seltenen Erden wies ein Versuch hị, der die Abscheidung eines kernisomeren Yttriums des bei der Uranspaltung auftretenden Isomerenpaares $\mathrm{Y}^{57} \mathrm{~d} \mathrm{Y}^{50} \mathrm{~min} \mathrm{Zr}^{10}$ zum Ziele hatte. Hierbei sollte mit $\overrightarrow{H i l f e}$ eines SzilardCha lmers - Verfahrens das durch den $\gamma$-Rückstoß gebildete $Y$-Isomer abgeschieden werden. $\mathrm{Zu}$ diesem Zweck wurde das in organischen Lösungsmitteln lösliche Yttrium-dibenzoylmethan dargestellt. Diese Verbindung erwies sich als völlig unbeständig gegen Wasser, da sie durch Hydrolyse augenblicklich in Dibenzoylmethan und Yttriumhydroxyd zerlegt wurde. Es lag also der Gedanke nahe, daß bei der Uranspaltung gebildete Seltene Erden sich ausschütteln lassen mußten, da sie mit dem Dibenzoylmethan keine gegen Wasser beständigen Komplexe bilden, wenn gleichzeitig die Verbindung des Uranylions mit dem Dibenzoylmethan sich als beständig gegen Wasser erwies. Dies war der Fall. Darüber hinaus bildet das Dibenzoylmethan mit den Elementen der 4. Gruppe und daher auch mit UX ebenfalls in organischen Lösungsmitteln lösliche und gegen Wasser recht beständige Komplexe. Blindversuche haben gezeigt, daß aus $10 \mathrm{~g}$ Uran im Gleichgewicht mit seinem Folgeprodukt, dem UX, von diesem nur der $10^{4}$. Teil ausschüttelbar ist. Das ist eine Aktivität, die bei Zählrohrmessungen etwa 100 Teilchen/Min. ausmacht und nicht mehr stört, da sie bei der Auswertung von Abfallskurven zum Schluß als konstant abgezogen werden kann. Ebenso wie das UX bleibt das bei der Bestrahlung entstandene Zirkon im organischen Lösungsmittel.

Gut ausschüttelbar hingegen sind alle die Elemente, die entweder keine beständigen Komplexe mit dem Dibenzoylmethan bilden oder deren Verbindungen mit diesen Diketonen leicht hydrolysieren. $\mathrm{Zu}$ diesen gehören insbesondere $Y$ Yttrium und die Seltenen Erden, aber auch gewisse $\mathrm{H}_{2} \mathrm{~S}$-fällbare Elemente, wie Antimon, Tellur, Molybdän sowie die Alkalien und Erdalkalien. Die Halogene bleiben ebenfalls in der organischen Phase, und auf Grund dieser Löslichkeit im organischen Lösungsmittel könnte angenommen werden, daß sie in atomarer Form entstehen.

Zur Darstellung des Uranyldibenzoylmethans gießt man die methylalkoholischen Lösungen von Uranylacetat

9 Naturwiss. 30, 107 [1942].

10 H. G ö t t e , Naturwiss. 29, 496 [1941]. 
und Dibenzoylmethan ${ }^{11}$ in der Kälte zusammen. Dabei tritt sofort eine intensive Rotgelbfärbung auf, und nach wenigen Sek. beginnt die Ausscheidung des Uranyldibenzoylmethans als Pulver, das aus viel heißem Äthylalkohol umkristallisiert werden kann. Andere Lösungsmittel eignen sich ebenfalls zur Reinigung, jedoch bildet das Uranyldibenzoylmethan mit fast allen Lösungsmitteln gut kristallisierende Additionsverbindungen, die z. Tl. prachtvoll orangerot sind, wie z. B. die des Acetons oder Essigesters.

Die Verglühungsanalyse einer aus Äthylalkohol umkristallisierten Probe ergab, daß abgesehen von $1 \mathrm{Mol}$. Alkohol 2 Mol. Dibenzoylmethan auf 1 Atom Uran kommen. Daher ist für den Uranyldibenzoylmethan-Komplex

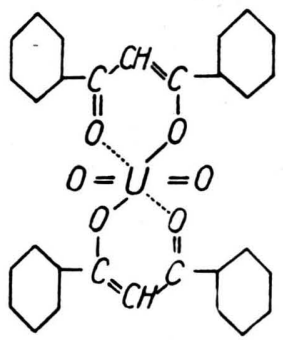

nebenstehende Formel anzunehmen. Das Uranyldibenzoylmethan ist eine orangerote, gut kristallisierende Substanz, die bei etwa $180^{\circ}$ Verfärbung zeigt und sich bei $245^{\circ} \mathrm{zu}$ zersetzen beginnt. Die Verbindung ist gut löslich in allen Ketonen und Estern, auch in Pyridin, nicht dagegen in reinen Kohlenwasserstoffen wie Benzol, Toluol, Xylol, Benzin usw.; auch Äthyläther löst schlecht. Äthylalkohol löst mittelmäßig, höhere Alkohole lösen schlechter. Die Innerkomplexverbindung ist zwar beständig gegen Wasser, wird aber von Säuren und Alkalien und auch schon von $\mathrm{NH}_{3}$ zersetzt. So kann man aus der Lösung von Uranyldibenzoylmethan in Essigester durch Schütteln mit verdünnter Säure in wenigen Sek. alles Uran aus der organischen in die wäßrige Phase überführen.

Eine andere Darstellungsmethode ist die folgende: Die Lösung von Dibenzoylmethan in Essigester oder einem anderen mit Wasser nicht mischbaren Keton wird mit einer wäßrigen Lösung von Uranylacetat geschüttelt. Dabei wandert das Uranylion in die organische Phase, und man gewinnt so eine Lösung des Urankomplexes, die gleich zur Bestrahlung verwendet werden kann ${ }^{12}$. Um alles Urån in die organische Lösung überzuführen, muß man den in der wäßrigen Phase entstehenden Säureüberschuß von Zeit zu Zeit mit $\mathrm{NH}_{3}$ neutralisieren. Diese Darstellungsmethode ist besonders dann am Platze, wenn es sich darum handelt, uran-X-freies Uranyldibenzoylmethan aus uran-X-freiem Uran zu gewinnen, weil sie in kurzer Zeit zum Ziele führt.

Zur Abscheidung der Seltenen Erden wird die bestrahlte Essigester-Lösung von Uranyldiben-

11 Leicht in zufriedenstellender Ausbeute darstellbar; vergl. C. W ey gand, Organ. chem. Experimentierkunst, Leipzig 1938.

12 Über das Verhalten des UX, also des Thoriums, bei diesem Verfahren wird in einer späteren Arbeit berichtet. zoylmethan mit Wasser geschüttelt. Die wäßrige Phase setzt sich in etwa $1 \mathrm{Min}$. ab; sie wird abgelassen und die $\mathrm{H}_{2} \mathrm{~S}$-Gruppe mit Hilfe eines Trägers gefällt. Dazu wurde 10-proz. salzsauer gemacht und als Träger Kupfer- oder Wismut- sowie Antimon- und Tellursalze zugegeben. Beim Einleiten des $\mathrm{H}_{2} \mathrm{~S}-\mathrm{Stromes}$ in die kochende Lösung wịd auf etwa $1 \% \mathrm{HCl}$ verdünnt. Gleichzeitig werden die Erdalkalien mit Bariumsulfat als Träger entfernt. Diese Niederschläge werden gemeinsam filtriert. Eine Wiederholung der beiden Fällungen entfernt die letzten Reste dieser Spaltprodukte. Im Filtrat sind in gewichtsloser Form Yttrium und die Seltenen Erden, das Element 93, die Alkalien und ein geringer Bruchteil (10 bis $20 \%$ ) des gebildeten Niobs. Auch Indium ist als Element der III. Gruppe wahrscheinlich ausschüttelbar. Nach Indium-Isotopen, die nur durch schnelle Neutronen gebildet werden, wurde aber nicht gesucht. Yttrium, Lanthan und die übrigen Seltenen Erden sowie das Element 93 und die geringe Niobaktivität werden mit einem Träger, z. B. Lanthan, als Hydroxyde oder als Oxalate niedergeschlagen, abfiltriert und können dann weiterverarbeitet werden. Hierbei kann man, um die Adsorption gebildeter Alkalien auszuschließen, 1 bis 2 mg Rubidium und Cäsium zusetzen. Die gesamten Operationen bis hierher von dem Ende der Bestrahlung an lassen sich bei einiger UUbung in 2 Min. ausführen. Hochspannungsanlage und Verarbeitungslaboratorium waren durch einen Weg von 3 Min. getrennt, so daß die Messung erst 5-6 Min. nach Ende der Bestrahlung beginnen konnte. So war es möglich, nach 10 Sek. Bestrahlung mit langsamen Neutronen die Existenz kurzlebiger Erden von 3 bis 5 Min. Halbwertszeit zu beweisen, ohne jedoch eine Trennung der Elemente dieser Gruppenfällung vornehmen zu können. Bei diesen Versuchen muß allerdings ein Dibenzoylmethan verwendet werden, das UXfrei ist, da das aus dem $\mathrm{UX}_{1}$ sich bildende Protaktinium-Isotop $\mathrm{UX}_{2}$ von $1 \mathrm{Min}$. Halbwertszeit nicht so beständige Dibenzoylmethankomplexe bildet und sich mit ausschütteln läßt. Im Blindversuch ist diese Aktivität sehr gut nachweisbar und kann so bei der Suche nach Seltenen Erden kurzlebige Isotope dieser Elementengruppe vortäuschen. Aus $10 \mathrm{~g}$ Uran lassen sich etwa 600 bis 800 Teilchen $\mathrm{UX}_{2} /$ Min. ausschütteln.

Die in Abb. 1 gezeichneten Kurven wurden durch Messung der Summenfällung der Erden gewonnen. Die Kurve I zeigt den Abfall eines Teilpräparates, 


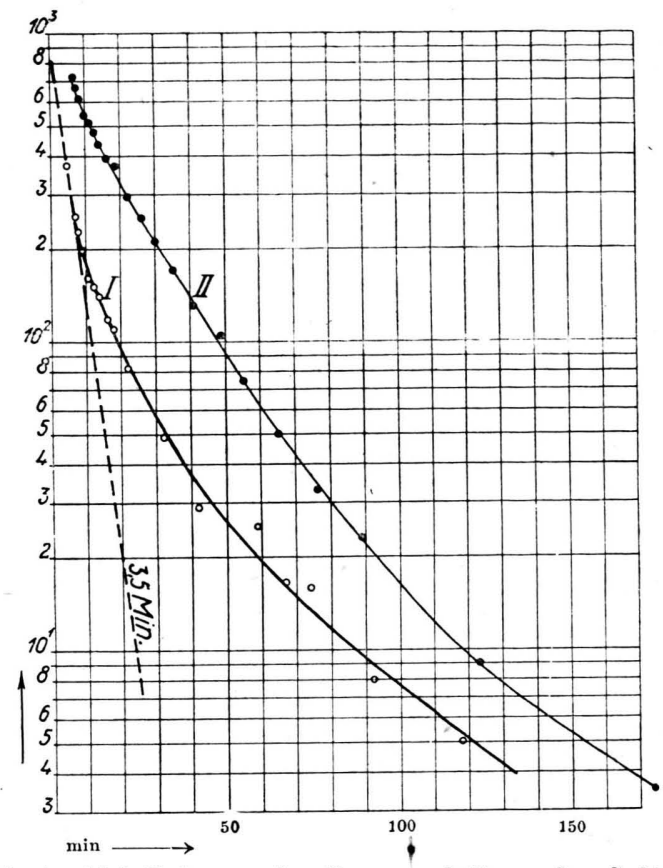

Abb. 1. Abfallskurve der Summenfällung der Seltenen Erden, gemessen 6 Min. nach Bestrahlungsende.

gemessen ohne Folie, während die Kurve II den Abfall einer stärkeren Aktivität, gemessen durch $3000 \mu \mathrm{Al}$, darstellt. Im zweiten Fall wird im wesentlichen die starke $\gamma$-Strahlung der kurzlebigen Seltenen Erden gezählt.

Bei langsamerer Verarbeitung nach einer Bestrahlung von 15 Min. konnte ein Cer-Isotop isoliert werden, dessen Aktivitätskurve, ohne Folie und durch $1000 \mu$ gemessen, die Abb. 2 zeigt. Dazu wurden die Summenfällung der Erden in Salzsäure gelöst, inaktives Rubidium und Cäsium sowie $2 \mathrm{mg}$ Cer und $100 \mathrm{mg}$ Lanthan hinzugegeben; dann wurde die Lösung auf 150 bis $200 \mathrm{ccm}$ verdünnt und nach Zugabe von Natriumperoxyd mit Natriumacetat versetzt und gekocht. Das ausgefallene 4-wertige Cer-Hydroxyd wurde erneut gelöst und unter denselben Bedingungen 2-mal umgefällt. Diese Umfällung des Cers ist erforderlich, da das ausfallende Cer(IV)-Hydroxyd immer Lanthan und damit Aktivitäten anderer Erden mitreißt. Durch Versuche mit aktiven Lanthan-Isotopen ließ sich zeigen, daß eine der CerMenge gleiche Lanthan-Menge bei dieser Art der Trennung mitfallen kann. Das gereinigte Cer konnte nach frühestens 11 bis 13 Min., vom Bestrahlungsende aus gerechnet, gemessen werden. Die Cer-Präparate wurden 1 bis 2 Min. nach ihrer Ausfällung unter den Zähler gebracht. Bei Messungen ohne Folie ergab sich die Kurve der Abb. 2.

Einem anfänglich flachen Abfall folgt ein stär. kerer, der schließlich in eine konstante Restaktivität übergeht. Wurde der Aktivitätsverlauf durch $1000 \mu \mathrm{Al}$ verfolgt, so ließ sich zunächst ein steiler Anstieg beobachten - Kurve II a, Abb. 2. Nach Überschreiten eines Maximums ging die Kurve ebenfalls in einen Abfall über, um schließlich in einer konstanten Aktivität zu enden. Diese Restaktivitäten sind auf bereits gebildete, langlebige Cer-Isotope, wie auch auf geringe UX-Aktivitäten zurückzuführen. Zieht man diese konstanten Aktivitäten von der gefundenen Cer-Kurve IIa ab, so erhält man die Kurve II b, die nach dem Maximum einen Abfall von etwa 30 Min. Halbwertszeit zeigt.

Wird das Cer nach einiger Zeit aufgelöst und eine erneute Lanthanabtrennung vorgenommen, so ist der Anstieg aufs neue, wenn auch geringer, zu beobachten, und das auch bei weiteren Abtrennungen in verschiedenen Zeitintervallen. Nach etwa 60 Min. bleibt er jedoch aus. Die abgetrennte Lanthanfällung zeigt nach Abzug der verbleibenden Restaktivitäten, die von bereits gebildeten längeren Isotopen und einer geringen Uran-X-Aktivität herrühren, eine Halbwertszeit von etwa 25 Min.

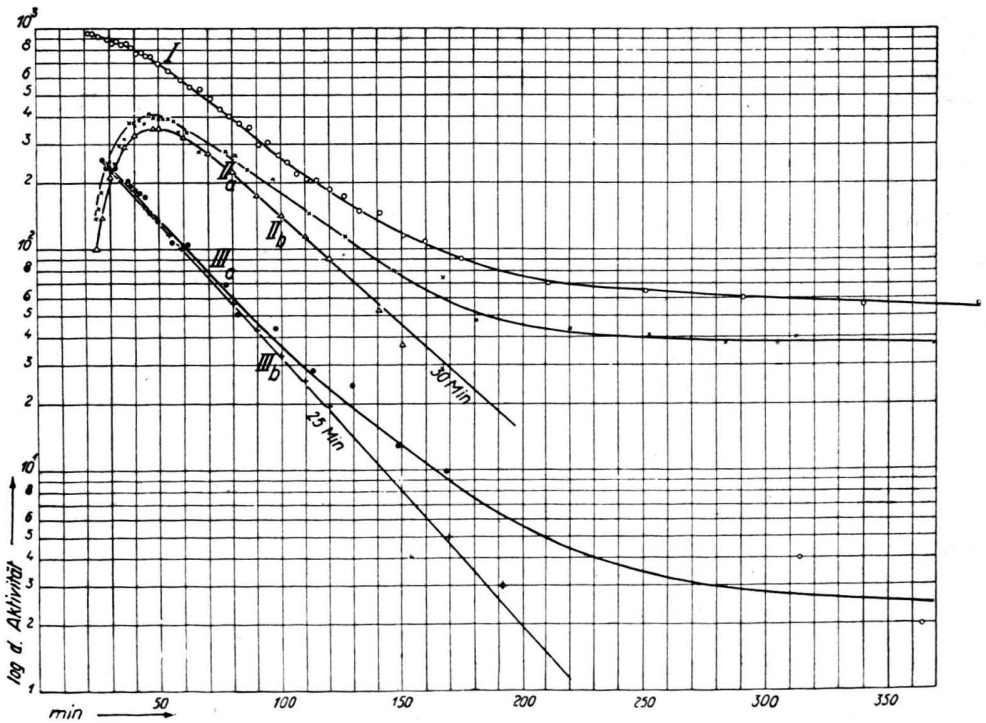

Abb. 2. Aktivitätskurven des Cer-Isotops, das ein Pr nachbildet. I. Gemessen ohne Folie, II. gemessen durch $1000 \mu \mathrm{Al}$, III. Abfallskurve des vom Cer abgetrennten Pr. 
(Kurve III a und III b Abb.2). Es liegt also die Nachbildung eines Folgeproduktes des Cers, nämlich eines Praseodyms von etwa 25 Min. Halbwertszeit vor, da ja bei der Cer-Lanthan-Trennung das Praseodym mit dem Lanthan zusammen abgeschieden wird. Aus dem endgültigen Ausbleiben des Anstiegs bei dem jeweils neu abgetrennten Cer nach etwa 50 bis 75 Min. kann man die Halbwertszeit dieses Isotops mit etwa 10 bis 15 Min. angeben. Der Verlauf der Cer-Kurve IIb in Abb.2 könnte zu der Annahme verleiten, es handele sich hier um ein Cer-Isotop, das ein im Vergleich zur Halbwertszeit des Cers kürzeres Praseodym nachbildet. Dann

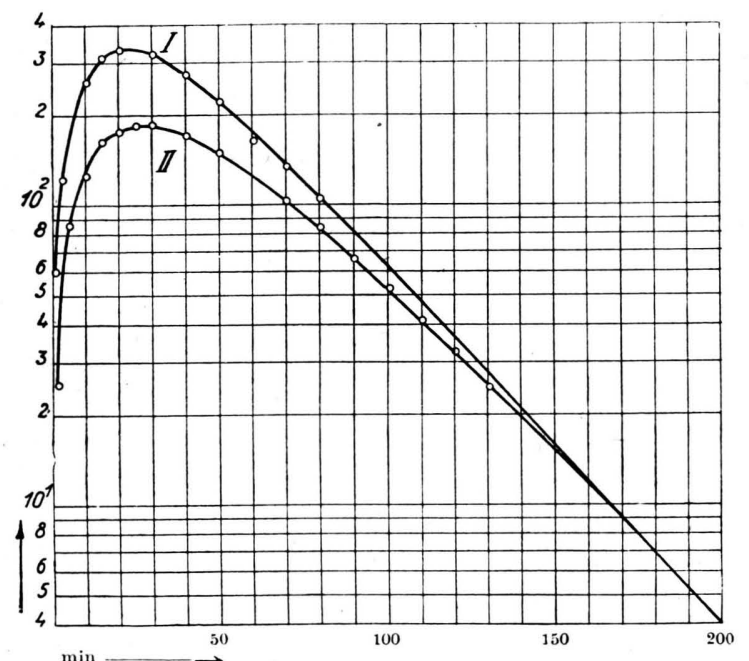

Abb. 3. Nachbildung eines Körpers mit 25 Min. Halbwertszeit aus einer Muttersubstanz von 10 Min.

(Kurve I) und 15 Min. (Kurve II).

müßte jedoch das abgetrennte Praseodym einen anfänglich steileren Abfall ergeben. Eine derartige steile Aktivitätsabnahme hat sich aber nie feststellen lassen. So erklärt sich dann der Kurvenverlauf durch die Nachbildung einer gegenüber der kurzlebigen Muttersubstanz etwas längerlebigen Tochtersubstanz. Die hierfür geltende Gleichung lautet:

$$
A=e^{-\lambda_{1} t}+\frac{\lambda_{1}}{\lambda_{2}-\lambda_{1}}\left(e^{-\lambda_{1} t}-e^{-\lambda_{2} t}\right)
$$

und die mit den ungefähr bestimmten Halbwertszeiten von 10 und 15 Min. für das Cer und 25 Min. für das Praseodym berechnete Kurve (Abb. 3) verläuft ähnlich der gefundenen Kurve II b (Abb.2). Das aus dem Cer nachgebildete Praseodym hat, wie der stärkere Anstieg der Nachbildungsaktivität bei der Messung durch $1000 \mu$ zeigt, eine härtere Strahlung als seine Muttersubstanz. Bei der
Messung durch $1000 \mu \mathrm{Al}$ wird die Cer-Strahlung unterdrückt, und nur das sich aus dem Cer nachbildende und abfallende Praseodym wird gemessen. Bei der angeführten Formel ist daher das erste Glied, das den Abfall des bei der Trennung vorhandenen Cers beschreibt, zu vernachlässigen.

Ein Versuch zur direkten Bestimmung der Halbwertszeit des Cers bei Verwendung von UX-haltigem Uranyldibenzoylmethan wurde wie folgt

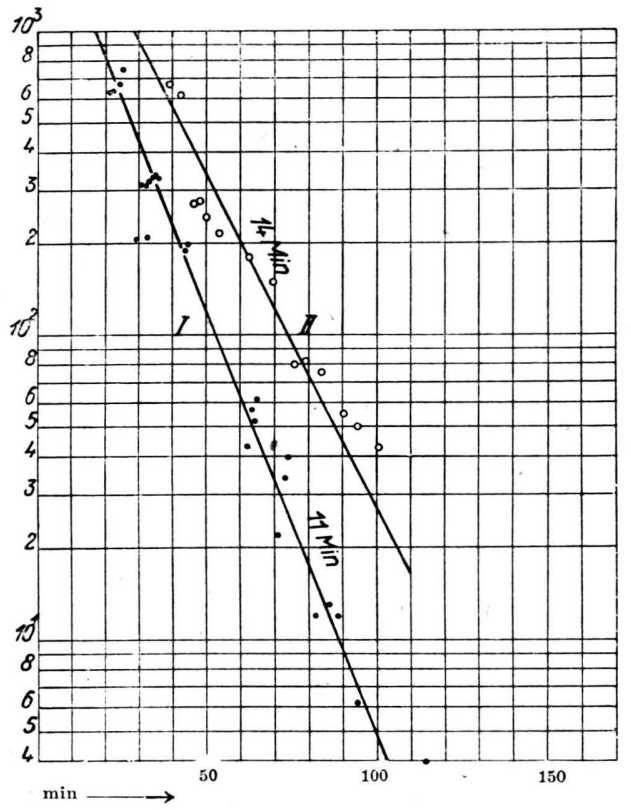

Abb. 4. Kurve I: Cer-Aktivität in Abständen von 10 Min. vom nachgebildeten Pr gereinigt. Kurve II: Pr in Abständen von 10 Min. am Cer abgeschieden.

durchgeführt. Das gereinigte Cer wird in Zeitabständen von je 10 Min. von seinem Folgeprodukt, dem Praseodym, befreit und dann jeweils durch $500 \mu \mathrm{Al}$ eine Zeitlang gemessen. Die so erhaltenen Aktivitätswerte zeigen ebenso wie die des jeweils abgetrennten Praseodyms die Abnahme des Cers. Es ergab sich eine Halbwertszeit von etwa 10 bis 15 Min. (Abb. 4).

Da jedoch bei den Cer-Fällungen nicht alles Uran X beim Cer zu bleiben scheint, sondern ein Teil mit dem Lanthan geht und sich die Gesamtanfangsaktivität aus Uran $\mathrm{X}+$ Cer zusammensetzt, wird durch die teilweise Abtrennung des Uran X eine Ungenauigkeit in den Kurvenverlauf getragen. Die genaue Bestimmung der Halbwertszeit durch Messung der Cer-Anfangsaktivitäten bei Verwendung von uran-X-freiem Uranyldibenzoylmethan ist nicht abgeschlossen worden. 
Es lag die Möglichkeit nahe, den Anstieg der Aktivität in den Kurven II a und II b auf Verunreinigungen, z. B. auf die Adsorption eines aus Tellur nachgebildeten Jods an das Cer-Hydroxyd zurückzuführen, ähnlich wie LaHydroxyd Jod adsorbiert. Dieses Jod konnte dann ein Xenon nachbilden. Diese Möglichkeit konnte ausgeschlossen werden, indem unter den oben beschriebenen Bedingungen eine Lanthanfällung blind, aber in Gegenwart von einer starken Aktivität des 26-Min.-Jods vorgenommen wurde. Es zeigte sich dabei, daß Jod unter den gegebenen Bedingungen so gut wie nicht adsorbiert wurde. Ubberdies hätte die Anstiegsaktivität im Falle der Jodadsorption ein neues Jodisotop bedeutet, das bei der gründlichen Erforschung der Jodisotope nicht zu erwarten wär. Die Nachbildung auf evtl. nicht vorher ausgefällte $\mathrm{H}_{2} \mathrm{~S}$-Elemente zurückzuführen, ist auch nicht möglich, da die letzte $\mathrm{H}_{2} \mathrm{~S}$-Gruppenfällung - es wurden mindestens 2 , häufig $3 \mathrm{H}_{2} \mathrm{~S}$-Fällungen vorgenommen vor der Messung immer einen anderen Aktivitätsabfall ergab und vor allem bereits bedeutend weniger Aktivität zeigte als das gemessene Cer. Auch nach sofortigem Wiederauflösen des Cer-Präparates wurden sowohl $\mathrm{H}_{2} \mathrm{~S}$ wie Barium-Fällungen vorgenommen, die sich als inaktiv erwiesen. Ebenso ließ sich zeigen, daß nicht etwa eine Niob-Aktivität an dem Aktivitätsanstieg beteiligt ist; dazu wurde das gereinigte Cer aufgelöst und mit einer Lösung von Niobpersäure in Schwefelsäure versetzt. Dann wurde das Niob mit Schwefeldioxyd ausgefällt und gemessen. Die sehr geringe Aktivität des Niobpräparates zeigte einen völlig anderen Abfall als die CerKurven.

Um festzustellen, welcher Bruchteil der Seltenen Erden durch Ausschütteln zu gewinnen ist, wurden gleich große Uranmengen in organischer und wäßriger Lösung bei gleicher Geometrie und gleicher Strahlenquelle gleiche Zeiten bestrahlt. Darauf wurde aus beiden Lösungen das 3,5-Stdn.-Yttrium abgeschieden und die Aktivitäten verglichen. Es ergab sich, daß etwa $80 \%$ des Yttriums bei zweimaligem Ausschütteln aus der organischen Phase zu gewinnen sind.

Nach derartigen Bestrahlungen ist die Lösung des Uranyldibenzoylmethans beliebig oft zur Abtrennung zu gebrauchen. Mehrmaliges Ausschütteln mit Wasser macht sie von den Spaltprodukten wieder frei, und jederzeit kann sie neu bestrahlt werden, so daß die Aufarbeitung des Urans entfällt. Jedoch ist darauf Rücksicht zu nehmen, daß bei der Verwendung von Essigester als Lösung'smittel für die Komplexverbindung des Urans bei jeder Ausschüttelung ein Teil dieses Lösungsmittels in der wäßrigen Phase gelöst wird. Dadurch steigt die Konzentration des Urans in der organischen Phase, und schließlich fällt Uranyldibenzoylmethan aus. Die Löslichkeit des Uranyldibenzoylmethans in Essigester beträgt etwa $25 \mathrm{mg} / \mathrm{cm}^{3}$. Aus diesem Grunde muß stets neuer Essigester zugeben werden. Ebenso ist es zweckmäßig, die organische Lösung von Zeit zu Zeit zu filtrieren, wenn Trübung durch nicht sich absetzende Wassertröpfchen oder dergl. eintritt.

Es empfiehlt sich auch, die Lösung vor der Bestrahlung nicht in dem zum Ausschütteln verwendeten Schütteltrichter aufzuheben, da ein Teil des gebildeten Uran X sich an der Glaswand des Trichters festsetzt und von dort je nach der Beschaffenheit des Glases mehr oder weniger stark mit herausgewaschen wird.

\title{
Über die Achsentemperatur der Niederstrombogensäule
}

\author{
Von KarL-Heinz Höcker
}

(Aus dem Kaiser-Wilhelm-Institut für Physik, Hechingen)

(Z. Naturforschg. 1, 382-384 [1946]; eingegangen am 3. Juni 1946)

\begin{abstract}
Es wird gezeigt, daß die Achsentemperatur einer Niederstrom-Entladung in der Umgebung solcher Temperaturen liegt, für die der ,,vollständige Wärmeleitungskoeffizient“ ein Minimum hat.
\end{abstract}

$\mathrm{I}^{\mathrm{n}}$ n der Theorie der Hochstrombogensäule ${ }^{1}$ haben wir den Verlauf eines ,vollständigen Wärmeleitungskoeffizienten" $K$ berechnet, der den Energieverlust hocherhitzter Luft an die Umgebung anzugeben gestattet. Für reinen Stickstoff wäre der Sachverhalt ähnlich, so daß die nachfolgenden Ausführungen auch hierfür gelten. Auf Grund der in Abb. 3 der zitierten Arbeit angegebenen Kurven wollen wir hier ein Minimumprinzip aussprechen,

1 K. H. Höcker u. W. Finkelnburg, Z. Naturforschg. 1, 305 [1946]. durch das die Achsentemperatur der Niederstrombogensäule festgelegt wird. Wir erläutern es an der Differentialgleichung der Lichtbogensäule

$$
-1 / r \cdot d / d r(r \cdot K d T / d r)=i E,
$$

die wir nach Multiplikation mit $r$ einmal integrieren können, wenn wir annehmen dürfen, daß infolge der Zylindersymmetrie die Feldstärke $E$ nicht vom Radius $r$ abhängt. Man erhält

$$
-r \cdot K \cdot d T / d r=I(r) \cdot E .
$$

